\title{
COVID-19 en niños y adolescentes mexicanos hasta el 10 de mayo de 2020. Enfoque en pacientes con diabetes
}

\section{COVID-19 in Mexican children and adolescents until May $10^{\text {th }}, 2020$. A focus on patients with diabetes}

\author{
Abril A. Arellano-Llamas ${ }^{1 *}$ y Álvaro Hernández-Caballero ${ }^{2}$
}

'Servicio de Endocrinología Pediátrica, Hospital General «Dr. Gaudencio González Garza»; ²Servicio de Hematología, Hospital de Especialidades «Dr. Antonio Fraga Mouret». Centro Médico Nacional La Raza del Instituto Mexicano del Seguro Social (IMSS), Ciudad de México, México

\section{RESUMEN}

\begin{abstract}
Antecedentes: La pandemia por enfermedad por coronavirus 2019 (COVID-19) afecta a la población de todas las edades. El gobierno de México ha publicado los datos epidemiológicos de los casos con prueba diagnóstica para el coronavirus 2 del síndrome respiratorio agudo grave (SARS-CoV-2). Objetivo: Conocer la epidemiología de la COVID-19 en personas de 0 a 18 años en México. Métodos: Análisis de la base de datos gubernamental con estadística descriptiva y razón de momios (RM). Resultados: 6,668 casos con edad de 0 a 18 años muestreados hasta el 10 de mayo de 2020 con letalidad del $1.75 \%$. La mortalidad se asoció con diagnóstico de neumonía (RM: 41.52; IC 95\%: 8.93-193.27), intubación (RM: 9.58; IC 95\%: 2.23-41.02), ingreso a terapia intensiva (RM: 2.74; IC 95\%: 0.65-11.44), inmunosupresión (RM: 1.09; IC 95\%: 1.001.029) y otra comorbilidad (RM: 8.77 ; IC 95\%: 2.54-30.52). La diabetes estuvo presente en 59 sujetos con edad de 12 años (0-16 años), con solo una muerte negativa para el SARS-CoV-2, sin asociación con mortalidad. No podemos asegurar la falta de asociación de muerte con obesidad en este grupo de edad. Conclusión: El comportamiento de la COVID-19 en los niños no se asocia con el sexo ni con el estado de diabetes para el desenlace de muerte. Hay riesgo de muerte cuando hay intubación, neumonía y/o inmunosupresión.
\end{abstract}

Palabras clave: COVID-19. Pediatría. Epidemiologia. México. Niños. Adolescentes.

\begin{abstract}
People of all ages are affected by coronavirus disease 2019 (COVID-19). The Mexican government have published the epidemiological data of patients tested for severe acute respiratory syndrome coronavirus 2 (SARS-CoV-2). Objective: To describe the epidemiology of Mexican population between 0-18 years with COVID-19. Methods: Descriptive statistics and OR of government epidemiological data. Results: We analyzed data from 6668 cases with ages from 0 to 18 years tested until May $10^{\text {th }}, 2020$. We found a lethality of $1.75 \%$. The mortality was associated with pneumonia (OR: 41.52; 95\% Cl: 8.93-193.27), intubation (OR: 9.58; CI 95\%: 2.23-41.02), admission to intensive care unit (OR: 2.74; 95\% Cl: 0.65-11.44), immunosuppression (OR: 1.09; 95\% $\mathrm{Cl}$ : 1.00-1.029), and other comorbidities (OR: 8.77; 95\% Cl: 2.54-30.52). Diabetes was present in 59 subjects with a median age of 12 years (0-16) and a death was reported in a negative SARS-CoV-2 diabetic patient; there were no association with mortality. We can't claim there is no association between mortality and obesity. Conclusions: The COVID-19 in pediatric population is not associated for death with sex or diabetes. There is greater death risk for intubation, pneumonia and immunosuppression.
\end{abstract}

Key words: COVID-19. Children. Adolescents. Pediatrics. Epidemiology. Mexico.
Correspondencia:

*Abril A. Arellano-Llamas

E-mail: abrilarellano@yahoo.com.mx
Fecha de recepción: 17-05-2020

Fecha de aceptación: 21-05-2020

DOI: 10.24875/RME.20000055
Disponible en internet: 11-06-2020 Rev Mex Endocrinol Metab Nutr. 2020;7:80-6

2462-4144 / @ 2020 Sociedad Mexicana de Nutricion y Endocrinologia, AC. Publicado por Permanyer. Éste es un artículo open access bajo la licencia CC BY-NC-ND (http://creativecommons.org/licenses/by-nc-nd/4.0/). 


\section{INTRODUCCIÓN}

El coronavirus 2 del síndrome respiratorio agudo grave (SARS-CoV-2) ocasiona la enfermedad por coronavirus 2019 (COVID-19), que puede provocar falla respiratoria aguda y alteraciones en otros sistemas del cuerpo humano, capaces de producir la muerte. Esta enfermedad infecciosa ha sido declarada pandemia por la Organización Mundial de la Salud y el primer caso en México se detectó en febrero de 2020¹.

El Gobierno de México ha hecho pública la base de datos de los casos sospechosos y confirmados de infección por SARS-CoV-2, incluyendo la información obtenida durante el estudio de caso epidemiológico. Estos datos se obtienen a través del Sistema de Vigilancia Epidemiológica de Enfermedades Virales, conformado por 475 unidades monitoras de enfermedad respiratoria viral del sector salud público y privado en todo el país ${ }^{2}$.

En la población general, la diabetes se ha reconocido como un factor de riesgo para el desarrollo de formas graves de la COVID-193. Esto ha desencadenado que se propongan protocolos especiales para continuar la atención de estos pacientes durante la epidemia utilizando estrategias como la telemedici$\mathrm{na}^{4}$. A la fecha se desconoce si la diabetes en la población pediátrica puede incrementar la incidencia de las formas moderadas o graves de la enfermedad y esto constituye un motivo de preocupación de los pacientes pediátricos y sus cuidadores primarios.

El objetivo de la presente investigación fue conocer la frecuencia de la COVID-19 en pacientes de 0 a 18 años, analizando la presencia de diabetes y otras comorbilidades como factores para el desarrollo de enfermedad grave o muerte, utilizando la base de datos abierta del Gobierno de México.

\section{MATERIAL Y MÉTODOS}

Se accedió a la base de datos abierta publicada por la Secretaría de Salud de México actualizada al 10 de mayo de $2020^{2}$. En el análisis se incluyeron todos los casos con una edad reportada de 0 a 18 años. Se obtuvo el dato de la edad reportado en años absolutos, el sexo (femenino o masculino), la información del estatus de intubado, neumonía, embarazo, diabetes, enfermedad pulmonar obstructiva crónica (EPOC), asma, inmunosupresión, hipertensión, otras comorbilidades, enfermedades cardiovasculares, obesidad, insuficiencia renal crónica, tabaquismo y el ingreso a terapia intensiva.

Para el análisis, la edad se dividió en menores o mayores de 10 años con la finalidad de considerar el inicio de la pubertad. Se codificó el estado de vivo o muerto por medio de la variable reportada como fecha de defunción.

Para esta publicación se analizaron los casos con diagnóstico positivo o negativo para SARS-CoV-2 y se omitieron los que se encontraban pendientes de diagnóstico.

El análisis estadístico incluyó la comparación de medias o medianas según la distribución de la muestra. Se compararon las variables cualitativas por chi cuadrada y los riesgos se estimaron por razón de momios (RM). Se consideró como significativa una $\mathrm{p}<0.05$.

\section{RESULTADOS}

En la base de datos se identificaron 7,494 casos entre cero y 18 años. Al 10 de mayo de 2020 se reportaron como positivos para SARS-CoV-2 un total de $682 \mathrm{ca}$ sos, como no positivos 5,986 casos y pendientes de resultado 826 casos. Para este análisis se utilizaron los datos de los 6,668 casos con diagnóstico definido, correspondientes al $89 \%$ de la muestra total. La tasa de positividad para el SARS-CoV-2 fue del 10.2\%. La mediana de la edad de los casos fue de 7 años (2-14 años). Doscientos pacientes se atendieron en hospitales privados, el resto se atendieron en el sistema público de salud. El $47.2 \%$ de los casos fueron mujeres. El $66.5 \%$ recibió atención de forma ambulatoria. Se reportaron 1,217 pacientes con neumonía (18.3\%) y 174 que requirieron intubación. Se registraron 70 menores embarazadas con muestra positiva para el SARS-CoV-2.

Dentro de las comorbilidades se registró una frecuencia de 59 personas con diabetes que representan el $0.9 \%$ de la población incluida, 13 personas con 
Tabla 1. Características generales de la población

\begin{tabular}{|c|c|c|c|c|}
\hline Variable & Casos totales & $\begin{array}{l}\text { SARS-CoV-2 } \\
\text { positivo }\end{array}$ & $\begin{array}{c}\text { SARS-CoV-2 } \\
\text { negativo }\end{array}$ & $\mathrm{p}$ \\
\hline $\mathrm{n}$ & 6,668 & 682 & 5,986 & \\
\hline Edad mediana años (rango intercuartílico) & $7(2,14)$ & $10.08(4-16)$ & $7.95(2-14)$ & $<0.001$ \\
\hline Sexo masculino $\mathrm{n}(\%)$ & $3,524(52.8)$ & $372(54.55)$ & $3,152(52.66)$ & 0.349 \\
\hline Intubado n (\%) & $174(2.6)$ & $10(1.74)$ & $164(2.74)$ & $<0.001$ \\
\hline Neumonía n (\%) & $1,217(18.3)$ & $82(12.02)$ & $1,135(18.96)$ & $<0.001$ \\
\hline Embarazo n (\%) & $70(1.0)$ & $10(1.47)$ & $60(1)$ & 0.469 \\
\hline Diabetes n (\%) & $59(0.9)$ & $17(2.49)$ & $42(0.70)$ & $<0.001$ \\
\hline Asma n (\%) & $323(4.8)$ & $20(2.96)$ & $303(5.06)$ & 0.046 \\
\hline Inmunosupresión n (\%) & $277(4.2)$ & $20(2.93)$ & $257(5.06)$ & 0.163 \\
\hline Hipertensión n (\%) & $60(0.9)$ & $10(1.47)$ & $50(0.84)$ & 0.097 \\
\hline Otra comorbilidad n (\%) & $477(7.2)$ & $40(5.87)$ & $437(7.30)$ & 0.255 \\
\hline Cardiovascular n (\%) & $148(2.2)$ & $11(1.61)$ & $137(2.29)$ & 0.402 \\
\hline Obesidad n (\%) & $188(2.8)$ & $39(5.72)$ & $149(2.49)$ & $<0.001$ \\
\hline Insuficiencia renal crónica n (\%) & $54(0.8)$ & $6(0.88)$ & $48(0.80)$ & 0.744 \\
\hline Más de una enfermedad concomitante n (\%) & $279(4.2)$ & $29(4.25)$ & $250(4.18)$ & 0.988 \\
\hline Tabaquismo n (\%) & $73(1.1)$ & $8(1.17)$ & $65(1.09)$ & 0.810 \\
\hline$\underline{\mathrm{UCI}} \mathrm{n}(\%)$ & $228(3.4)$ & $16(2.35)$ & $212(3.54)$ & $<0.001$ \\
\hline Estado muerto n (\%) & $109(1.6)$ & $12(1.76)$ & $97(1.62)$ & 0.786 \\
\hline
\end{tabular}

SARS-CoV-2: coronavirus 2 del síndrome respiratorio agudo grave; UCI: unidad de cuidados intensivos.

EPOC (0.2\%), 323 con asma (4.8\%), 277 con inmunosupresión (4.2\%), 60 sujetos hipertensos $(0.9 \%)$ y 477 personas con otra comorbilidad no especificada (7.2\%). Se reportó enfermedad cardiovascular en 148 personas $(2.2 \%)$, obesidad en 188 sujetos $(2.8 \%)$, enfermedad renal crónica en 54 personas $(0.8 \%)$ y tabaquismo en 73 personas (1.1\%). El detalle de los datos se muestra en la tabla 1.

Dentro del estudio epidemiológico se reportó contacto con otro caso con COVID-19 en el $27.2 \%$ de los casos. Finalmente se reportaron ingresados en terapia intensiva de 228 personas, que representan el $3.4 \%$ de la población en estudio. En los casos reportados como positivos se observó una letalidad del $1.75 \%$. Las características de los pacientes finados se describen en la tabla 2, y resaltan por su frecuencia: diabetes, ingreso a terapia intensiva, hipertensión, neumonía e intubación.

En los casos positivos para SARS-CoV-2 se incrementó el riesgo de muerte al requerir intubación, ingreso a la unidad de cuidados intensivos, diagnóstico de neumonía, el antecedente de inmunosupresión u otra comorbilidad (Tabla 3). En los casos con antecedentes de diabetes, asma, insuficiencia renal, hipertensión, obesidad y tabaquismo no se registraron muertes hasta la fecha de análisis de este estudio.

De los sujetos analizados se conocía el antecedente de diabetes en el $98.2 \%$ de los casos, pues se reportó como "se ignora» en 14 sujetos. La mediana de edad de los 59 casos con diabetes fue de 12 años (0-16). Como comorbilidad adicional a la diabetes se reportó la existencia de insuficiencia renal crónica en tres personas y la coexistencia de obesidad en 16. Hubo solo una muerte registrada en el grupo de pacientes con diabetes y resultó negativo en la prueba para SARS-CoV-2. El detalle de los resultados en población que vive con diabetes se muestra en la tabla 4.

\section{DISCUSIÓN}

Los coronavirus usualmente causan enfermedades del tracto respiratorio superior. En las últimas dos 
Tabla 2. Características de los pacientes que fallecieron

\begin{tabular}{|c|c|c|c|}
\hline Variable & $\begin{array}{c}\text { SARS-CoV-2 positivo } \\
\mathrm{n}=12\end{array}$ & $\begin{array}{c}\text { SARS-CoV-2 negativo } \\
\mathrm{n}=97\end{array}$ & $\mathrm{p}$ \\
\hline Edad años mediana (rango intercuartílico) & $4.67(1-8)$ & $5.66(1-10)$ & 0.894 \\
\hline Sexo masculino n (\%) & $8(66.7)$ & $51(52.6)$ & 0.355 \\
\hline Sexo femenino $\mathrm{n}(\%)$ & $4(33.3)$ & $46(47.4)$ & \\
\hline Diabetes n (\%) & $0(0)$ & $1(1)$ & 0.826 \\
\hline Hipertensión n (\%) & $0(0)$ & $3(3.1)$ & 0.675 \\
\hline Obesidad n (\%) & $0(0)$ & $2(2.1)$ & 0.826 \\
\hline Múltiples patologías (más de una) n (\%) & $3(25)$ & $17(17.5)$ & 0.215 \\
\hline Neumonía n (\%) & $10(83.3)$ & $63(64.9)$ & 0.201 \\
\hline Intubado n (\%) & $4(33.3)$ & $32(33)$ & 0.719 \\
\hline Estancia en UCI n (\%) & $3(25)$ & $23(23.7)$ & 0.723 \\
\hline
\end{tabular}

SARS-CoV-2: coronavirus 2 del síndrome respiratorio agudo grave; UCl: unidad de cuidados intensivos.

Tabla 3. Características de pacientes con prueba positiva para SARS-CoV-2

\begin{tabular}{|c|c|c|c|c|}
\hline Variable & $\begin{array}{c}\text { Vivo } \\
\mathrm{n}=670\end{array}$ & $\begin{array}{c}\text { Muerto } \\
\mathrm{n}=12\end{array}$ & RM (IC 95\%) & $\mathrm{p}$ \\
\hline Edad < 10 años n (\%) & $282(42.1)$ & $9(75)$ & $2.206(0.568-8.566)$ & 0.243 \\
\hline Sexo mujer n (\%) & $306(45.7)$ & $4(33.3)$ & $0.594(0.177-1.99)$ & 0.396 \\
\hline Intubado n (\%) & $6(0.9)$ & $4(33)$ & $9.583(2.239-41.020)$ & $0.001^{*}$ \\
\hline Neumonía n (\%) & $72(10.7)$ & $10(83.3)$ & $41.52(8.93-193.27)$ & $<0.001$ \\
\hline Diabetes n (\%) & $17(2.5)$ & $0(0)$ & & 0.576 \\
\hline Asma n (\%) & $20(3)$ & $0(0)$ & & 0.543 \\
\hline Inmunosupresión n (\%) & $17(2.5)$ & $3(25)$ & $1.019(1.008-1.029)$ & $0.0001^{*}$ \\
\hline Hipertensión n (\%) & $10(1.5)$ & $0(0)$ & & 0.669 \\
\hline Otra comorbilidad n (\%) & $36(5.4)$ & $4(33.3)$ & $8.778(2.524-30.525)$ & $0.0001^{*}$ \\
\hline Cardiovascular n (\%) & $10(1.5)$ & $1(8.3)$ & $5.98(0.704-50.855)$ & 0.063 \\
\hline Obesidad n (\%) & $39(5.8)$ & $0(0)$ & & 0.388 \\
\hline Insuficiencia renal crónica n (\%) & $6(0.9)$ & $0(0)$ & & 0.703 \\
\hline Patología múltiple n (\%) & $26(3.9)$ & $3(25)$ & $8.25(2.11-32.304)$ & $<0.001^{*}$ \\
\hline Tabaquismo n (\%) & $8(1.2)$ & $0(0)$ & & 0.649 \\
\hline $\mathrm{UCI} n(\%)$ & $13(1.9)$ & $3(25)$ & $2.744(0.658-11.440)$ & 0.152 \\
\hline
\end{tabular}

*Estadísticamente significativo.

SARS-CoV-2: coronavirus 2 del síndrome respiratorio agudo grave; UCl: unidad de cuidados intensivos; RM: razón de momios; IC: intervalo de confianza.

décadas los coronavirus del síndrome respiratorio de oriente medio y el SARS-CoV-1 han ocasionado una infección más grave que evoluciona a neumonía y puede ocasionar la muerte. Desde diciembre de 2019 la pandemia de COVID-19 ocasionada por el SARS-CoV-2 se ha esparcido por el mundo, ocasionando al menos 283,732 muertes hasta el 10 de mayo de $2020^{5}$.

En la COVID-19 la edad avanzada juega un papel importante en determinar el riesgo de formas graves y letales de la enfermedad ${ }^{5}$, pero aun en la población pediátrica es posible observar casos graves que terminan en la muerte. En el grupo de edad de 0 a 18 años se observó una letalidad del 1.75\%, la cual resultó significativamente menor a la de la población general en México. Sin embargo, es mayor a la que se reportó en la provincia de Hubei, en China, donde se registró un fallecimiento en 1,407 casos confirmados, lo que equivale al $0.07 \%$. En la serie de casos de China no se describe la prevalencia de comorbilidades que tal vez podrían explicar 
Tabla 4. Características de los pacientes con diabetes

\begin{tabular}{|c|c|c|c|c|}
\hline Variable & $\begin{array}{l}\text { Todo el grupo } \\
\qquad \mathrm{n}=59\end{array}$ & $\begin{array}{c}\text { SARS-CoV-2 } \\
\text { positivo } \\
n=17\end{array}$ & $\begin{array}{c}\text { SARS-CoV-2 } \\
\text { negativo } n=42\end{array}$ & $\mathrm{p}$ \\
\hline Edad años (mediana) & $12(0,16)$ & $10(0,17)$ & $12(3,15)$ & 0.872 \\
\hline Edad < 10 años n (\%) & $23(38.9)$ & $8(47.1)$ & $15(35.7)$ & 0.418 \\
\hline Edad > 10 años n (\%) & $36(61.1)$ & $9(52.9)$ & $27(64.3)$ & \\
\hline Sexo hombre n (\%) & $24(40.6)$ & $5(29.4)$ & $19(45.2)$ & 0.262 \\
\hline Sexo mujer n (\%) & $35(59.4)$ & $12(70.6)$ & $23(54.8)$ & \\
\hline Intubado n (\%) & $3(5)$ & $1(5.9)$ & $2(4.8)$ & 0.689 \\
\hline Neumonía n (\%) & $21(35.6)$ & $11(64.7)$ & $10(23.8)$ & 0.003 \\
\hline Paciente hospitalizado n (\%) & $33(55.9)$ & $11(64.7)$ & $22(52.4)$ & 0.388 \\
\hline Embarazo n & $0(0)$ & $0(0)$ & $0(0)$ & \\
\hline Hipertensión n (\%) & $12(20.3)$ & $5(29.4)$ & $7(16.7)$ & 0.271 \\
\hline Cardiovascular n (\%) & $5(8.4)$ & $0(0)$ & $5(11.9)$ & 0.137 \\
\hline Obesidad n (\%) & $16(27.1)$ & $3(17.6)$ & $13(31)$ & 0.298 \\
\hline Insuficiencia renal crónica n (\%) & $3(5)$ & $0(0)$ & $3(7.1)$ & 0.258 \\
\hline Más de una comorbilidad n (\%) & $30(50.8)$ & $7(41.2)$ & $23(54.8)$ & 0.344 \\
\hline UCI n (\%) & $3(5)$ & $0(0)$ & $3(7.1)$ & 0.282 \\
\hline Muertos n (\%) & $1(1.6)$ & $0(0)$ & $1(2.4)$ & 0.521 \\
\hline
\end{tabular}

SARS-CoV-2: coronavirus 2 del síndrome respiratorio agudo grave; UCl: unidad de cuidados intensivos.

las diferencias en la mortalidad con la población mexicana. En población pediátrica de México, como en los adultos, el requerimiento de intubación se traduce en un mal pronóstico ${ }^{6}$.

Resulta relevante que en este grupo de edad la mayoría de los pacientes con diagnóstico de neumonía o que requirieron intubación tuvieron una prueba negativa para SARS-CoV-2. Esto sugiere que en niños y adolescentes es necesario excluir agentes infecciosos diversos como influenza o bacterias y considerar la cobertura con antibióticos en pacientes con neumonía y sospecha de COVID-19.

Es notable que en la población pediátrica no se observó una mayor gravedad en el sexo masculino, a diferencia de otras series en adultos, lo que sugiere fuertemente que la exposición prolongada a las hormonas masculinas puede modificar el riesgo a formas más graves de COVID-197. También se ha postulado que la señalización de los receptores de estrógenos puede tener un efecto protector en las mujeres $^{8}$.

La prevalencia de obesidad del $2.8 \%$ en esta base de datos es menor a la reportada en la encuesta nacional de salud para este grupo, del $12.8 \%{ }^{9}$. Por la naturaleza de los datos, se desconoce si el diagnóstico de obesidad se basó en antropometría o por el antecedente referido en el interrogatorio, y tampoco se conoce el grado de obesidad o sobrepeso. Se ha reportado previamente que los pacientes o los padres pueden subestimar el estado de sobrepeso u obesidad al interrogatorio ${ }^{10}$, por lo que esto pudo haber influido en un subregistro de esta comorbilidad, y por lo tanto no descarta la posibilidad de asociaciones de la obesidad con desenlaces adversos en este grupo de pacientes.

Un dato de importancia consiste en el reporte de tabaquismo en una población de menores de edad. Se ha demostrado que el tabaquismo incrementa la morbilidad y la mortalidad prematura. Además, la población joven se encuentra expuesta a formas más adictivas del uso de tabaco, como los cigarrillos electrónicos y el vapeo ${ }^{11}$.

En el grupo de pacientes con diabetes, la mediana de edad fue de 12 años (0-16 años), lo que sugiere fuertemente que la mayoría de estos casos pueden corresponder a diabetes tipo 1. Es de resaltar la presencia de enfermedad renal crónica como comorbilidad 
en edades tempranas, lo que podría traducir en un mal control metabólico de esta población joven. También sobresale la existencia de otras patologías asociadas como la obesidad, aunque por los datos disponibles no se puede diferenciar si estos pacientes cursaban con diabetes tipo 2 . Se hace manifiesta la necesidad de mejorar el control metabólico a fin de disminuir la incidencia de desenlaces desfavorables agudos o crónicos ${ }^{12}$.

En los datos analizados, la presencia de diabetes no se asoció con mortalidad por COVID-19. En un metaanálisis que incluyó datos de 33 estudios y 16,003 pacientes, la diabetes estuvo asociada con una duplicación del riesgo de muerte y gravedad, en comparación con la población sin diabetes ${ }^{13}$, esto evidencia la necesidad de continuar la vigilancia de los pacientes con COVID-19 y antecedente de diabetes. Es posible que el tiempo de evolución de la diabetes también juegue un papel para determinar esta diferencia en el desenlace a diferencia de los adultos; tampoco debe menospreciarse que a este grupo de pacientes, en la mayoría de los casos, les atienden especialistas endocrinólogos pediatras y se cuenta con cuidadores primarios que mejoran el control metabólico en la etapa preadolescente. Otra explicación para la diferencia de la asociación de diabetes y muerte respecto los adultos es que quizá en este grupo estemos observando diabetes tipo 1 más representada que la tipo 2; en este caso la fisiopatología de la enfermedad es diferente, y en los adultos la enfermedad cardiovascular (incluida la hipertensión) ha sido asociada fuertemente con un mal desenlace, ambas situaciones fuertemente correlacionadas con el tipo 2 de la diabetes y con un requerimiento de exposición temporal mayor para la tipo $1^{14}$.

Se reconoce que la población con diabetes es un grupo vulnerable para complicaciones por COVID-193. Durante una pandemia es un reto mantener el control metabólico, ya que se dificulta contar con visitas médicas frecuentes, acceso a medicamentos y estudios de laboratorio. Además, los pacientes sufren cambios importantes en la rutina de alimentación y actividad física derivado de la cuarentena.

El exceso de pacientes SARS-COV-2 negativos para complicaciones puede corresponder a un muestreo aumentado en los niños que tenían estados de mayor gravedad, además en la base de datos gubernamental se incluyen pacientes que aún corresponden con la oleada de influenza estacional. No se descarta la posibilidad de que en este grupo se incluyeran pacientes con resultado falsamente negativo.

Hasta la fecha no hay manejo específico para la COVID-19 en el grupo de pacientes pediátricos, no hay ensayos clínicos que sustenten manejos, dosis o recomendaciones especiales ${ }^{15}$.

El origen de los datos de esta investigación puede tener limitantes. Debido a que solo se incluyen a las personas a quienes se les realizó la prueba de reacción en cadena de la polimerasa para SARS-COV-2 es posible que haya una mayor representación de los casos graves. No ha sido posible corroborar la metodología para establecer el diagnóstico de las comorbilidades ni se cuenta con los datos de su gravedad. La base de datos no cuenta con variables clínicas o de laboratorios que permita corroborar los diagnósticos. Las autoridades reconocen que los datos de estas bases son preliminares y están sujetos a validación.

Consideramos de gran valor científico el contar con bases de datos epidemiológicos en tiempo real durante la pandemia de COVID-19. Esto permite estudiar el comportamiento de poblaciones específicas y diseñar políticas para estos grupos de pacientes. Seguramente estos datos serán objeto de mayor investigación por la comunidad científica.

\section{FINANCIAMIENTO}

La presente investigación no ha recibido ayudas específicas provenientes de agencias del sector público, sector comercial o entidades sin ánimo de lucro.

\section{CONFLICTO DE INTERESES}

Los autores declaran no tener conflicto de intereses. 
RESPONSABILIDADES ÉTICAS

Protección de personas y animales. Los autores declaran que para esta investigación no se han realizado experimentos en seres humanos ni en animales.

Confidencialidad de los datos. Los autores declaran que han seguido los protocolos de su centro de trabajo sobre la publicación de datos de pacientes.

Derecho a la privacidad y consentimiento informado. Los autores declaran que en este artículo no aparecen datos de pacientes.

\section{BIBLIOGRAFÍA}

1. Hernández-Ávila M, Alpuche-Aranda CM. Mexico: Lessons learned from the 2009 pandemic that help us fight COVID-19. Healthc Manage Forum. Healthc Manage Forum. 2020;33(4):158-63.

2. Información referente a casos COVID-19 en México [Internet]. México: Gobierno de México, Secretaría de Salud [fecha de consulta: 12 de mayo de 2020]. Disponible en: https://datos.gob.mx/busca/dataset/informacion-referente-a-casos-covid-19-en-mexico

3. Katulanda P, Dissanayake HA, Ranathunga I, Ratnasamy V, Wijewickrama PSA, Yogendranathan N, et al. Prevention and management of COVID-19 among patients with diabetes: an appraisal of the literature. Diabetologia. 2020 May 14:1-13.
4. Garg SK, Rodbard D, Hirsch IB, Forlenza GP. Managing new-onset type 1 diabetes during the COVID-19 pandemic: Challenges and opportunities. Diabetes Technol Ther. 2020 Apr 17. doi: 10.1089/dia.2020.0161. Online ahead of print.

5. Roser M. Ritchie H, Ortiz-Ospina E, Hasell J. Coronavirus (COVID-19) deaths [Internet]. EE.UU.: Our World in Data [fecha de consulta: 13 de mayo de 2020]. Disponible en: https://ourworldindata.org/covid-deaths

6. Zhou F, Yu T, Du R, Fan G, Liu Y, Liu Z, et al. Clinical course and risk factors for mortality of adult inpatients with COVID-19 in Wuhan, China: a retrospective cohort study. Lancet. 2020;395(10229):1054-62.

7. Sharma G, Volgman AS, Michos ED. Sex differences in mortality from COVID-19 pandemic: Are men vulnerable and women protected? JACC Case Rep. 2020 May 4. doi: 10.1016/j.jaccas.2020.04.027. Online ahead of print.

8. Channappanavar R, Fett C, Mack M, Ten Eyck PP, Meyerholz DK, Perlman S. Sex-based differences in susceptibility to severe acute respiratory syndrome coronavirus infection. J Immunol Baltim Md 1950. 2017; 198(10):4046-53.

9. Encuesta Nacional de Salud y Nutrición de Medio Camino 2016 [Internet]. México: Gobierno de México [fecha de consulta: 13 de mayo de 2020]. Disponible en: http://www.gob.mx/salud/documentos/encuestanacional-de-salud-y-nutricion-de-medio-camino-2016

10. Arellano-Llamas AA, Garrido-Magaña E (dir). Satisfacción con la imagen corporalen adolescentes de una escuela secundaria del sur del Distrito Federal. [tesis] [México]: Universidad Nacional Autónoma de México. 2012. Disponible tesis.unam.mx

11. US Preventive Services Task Force, Owens DK, Davidson KW, Krist AH, Barry MJ, Cabana M, Caughey AB, et al. Primary care interventions for prevention and cessation of tobacco use in children and adolescents: US Preventive Services Task Force Recommendation Statement. JAMA. 2020 28;323(16):1590-8.

12. Dong $Y, M o X, H u Y$, Qi $X$, Jiang F, Jiang Z, et al. Epidemiology of COVID-19 among children in China. Pediatrics. Pediatrics. 2020 Mar 16:e20200702. doi: 10.1542/peds.2020-0702. Online ahead of print.

13. Kumar A, Arora A, Sharma P, Anikhindi SA, Bansal N, Singla V, et al. Is diabetes mellitus associated with mortality and severity of COVID-19? A meta-analysis. Diabetes Metab Syndr. 2020;14(4):535-45.

14. Rochlani Y, Pothineni NV, Kovelamudi S, Mehta JL. Metabolic syndrome: pathophysiology, management, and modulation by natural compounds. Ther Adv Cardiovasc Dis. 2017;11(8):215-25.

15. Carlotti AP de CP, Carvalho WB de, Johnston C, Rodriguez IS, Delgado AF. COVID-19 diagnostic and management protocol for pediatric patients. Clinics (Sao Paulo). 2020 Apr 17;75:e1894. 